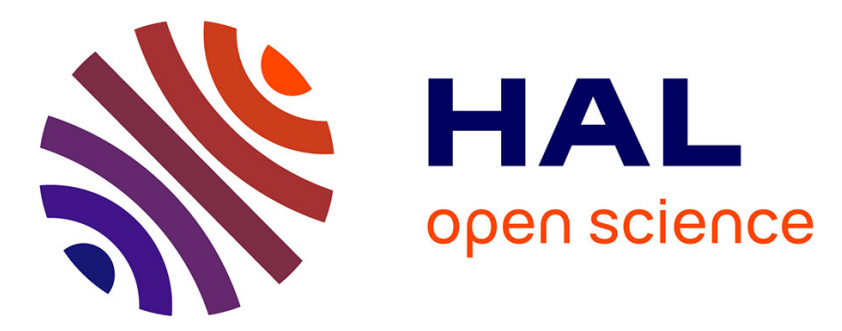

\title{
Uncertainty analysis of a 2-DOF planar parallel robot by means of fuzzy dynamic approach
}

\author{
Fabian Andres Lara-Molina, Didier Dumur, E.H. Koroishi
}

\section{To cite this version:}

Fabian Andres Lara-Molina, Didier Dumur, E.H. Koroishi. Uncertainty analysis of a 2-DOF planar parallel robot by means of fuzzy dynamic approach. Proceedings 13th Latin American Robotics Symposium and 4th Brazilian Symposium on Robotics LARS/SBR, Oct 2016, Recife, Brazil. pp. 275-280, 10.1109/LARS-SBR.2016.53 . hal-01644758

\section{HAL Id: hal-01644758 \\ https://hal-centralesupelec.archives-ouvertes.fr/hal-01644758}

Submitted on 12 Mar 2020

HAL is a multi-disciplinary open access archive for the deposit and dissemination of scientific research documents, whether they are published or not. The documents may come from teaching and research institutions in France or abroad, or from public or private research centers.
L'archive ouverte pluridisciplinaire HAL, est destinée au dépôt et à la diffusion de documents scientifiques de niveau recherche, publiés ou non, émanant des établissements d'enseignement et de recherche français ou étrangers, des laboratoires publics ou privés. 


\title{
Uncertainty Analysis of a 2-DOF Planar Parallel Robot by means of Fuzzy Dynamic Approach
}

\author{
F. A. Lara-Molina ${ }^{1}$, D. Dumur ${ }^{2}$ and E. H Koroishi ${ }^{1}$
}

\begin{abstract}
This paper aims at analyzing the effect of uncertain friction in the active joints of a 2-DOF planar parallel robot using a fuzzy dynamic approach. The uncertain parameters of friction are modeled as fuzzy variables and the dynamic response of the robot is computed by using fuzzy dynamic analysis. The dynamics of the robot under uncertain friction including a computed torque position controller is analyzed. Numerical simulations illustrate the proposed methodology in order to describe and quantify the effect of uncertain frictions on the dynamic performance of the parallel robot.
\end{abstract}

\section{INTRODUCTION}

Parallel robots are unavoidably subject to uncertainties. The main sources of uncertainties include various aspects such as manufacturing limitations and assembling tolerances of the mechanical parts, noise in the sensors, and unmodeled dynamics in the control system. Furthermore, in several applications, the robots operate with different values of payload to perform a specific task (e.g. pick and place robots).

Despite uncertainties, the parallel robots should be able to execute diverse tasks with high accuracy and repeatability which requires high reliability (e.g. robots used in medical applications). Therefore it is necessary to analyze the effects of uncertain parameters on the dynamic response in order to observe the behavior of the parallel robots under these conditions.

Several methodologies have been used to analyze uncertainties in robot manipulators. The stochastic approach has been widely applied to study the effects of uncertain parameters on the behavior of robot manipulators. In agreement with this approach, the effect of tolerances associated with the manipulator parameters on the reliability was studied [1], [2]. Moreover, Polynomial Chaos Theory was applied to study the effect of uncertain inertia and payload on SCARA robot dynamics [3].

Additionally, an approach based on fuzzy dynamic analysis has been applied to study uncertain parameters in a two-link planar robot manipulator [4]. The aforementioned approach is suitable when the stochastic process that governs the uncertainty is unknown; thus uncertain parameters are modeled by means of fuzzy variables [5]. This contribution aims at applying the fuzzy dynamic analysis in order to analyze and quantify the effect of uncertain parameters

${ }^{1}$ F. A. Lara Molina is with the Department of Mechanical Engineering, Federal University of Technology - Paraná, Cornélio Procópio - PR, Brazil fabianmolinalutfpr.edu.br

${ }^{2} \mathrm{D}$. Dumur is with Laboratoire des signaux et Systèmes, CentraleSupélec-CNRS-Université Paris-Sud-Université ParisSaclay, Control Department, 91192 Gif sur Yvette cedex, France Didier.Dumurecentralesupelec.fr modeled as fuzzy variables in the dynamic response of parallel robots, specifically, a 2-DOF planar parallel robot.

According to the previous discussion, it is necessary to analyze the dynamic response of parallel robots under uncertain parameters, i.e., to analyze how the robot dynamics is affected by uncertain friction in the joints, and thus to quantify these effects into the dynamic response of the robot by using straightforward numerical methods. Moreover, it is necessary to evaluate the effect of these uncertainties in terms of position accuracy of the parallel robots.

In this contribution, the dynamics of a 2-DOF planar parallel robot with fuzzy uncertain friction is analyzed. The simulation of the robot under uncertainties is performed by means of a numerical method based on fuzzy dynamic analysis. A computed torque control scheme is analyzed under fuzzy uncertainties. The remainder of this paper is organized in five sections sections. After the introduction, section II introduces the parallel robot model and the tracking position control scheme. In section III, the fuzzy dynamic analysis is presented. The numerical results are shown in section IV. Finally, the conclusions and further work are outlined.

\section{ROBOT MODELING}

The 2-DOF planar parallel robot has two kinematic chains. Each kinematic chain includes an active or actuated joints, denoted as $\theta_{a i}$, a passive or free joint, denoted as $\theta_{p i}$ for $i=1,2$, and two links. The geometry planar parallel robot is defined according to Fig. 1. The active joints are rotational and they are located at points $A_{i}$ with Cartesian coordinates $\left(x_{a_{i}}, 0\right)^{T}$, for $i=1,2$. The passive joints are rotational and they are located at the points $B_{i}$. The length of the links are defined by $l_{11}, l_{12}, l_{21}$ and $l_{22}$. The end effector of the mechanism is located at $P$ where its position is defined by the Cartesian coordinates $\left(x_{p}, y_{p}\right)$. Additionally, the fixed reference frame $O$ is defined in the middle of $A_{1} A_{2}$. The gravity acceleration acts perpendicular to the plane $x y$ within the manipulator works.

\section{A. Dynamic of the Kinematic Chain}

The modeling of each single kinematic chain is analyzed separately in order to simplify the total formulation of the dynamics of the robot. The dynamic equation is obtained by means of the Lagrange formulation presented in the Eq. (1). This approach takes into account the kinetic and potential energies of the mechanism according to [6].

$$
\boldsymbol{\tau}_{i}-\mathbf{f}_{i}=\frac{d}{d t}\left(\frac{\partial L_{i}}{\partial \dot{\boldsymbol{\theta}}_{i}}\right)-\frac{\partial L_{i}}{\partial \boldsymbol{\theta}_{i}}
$$




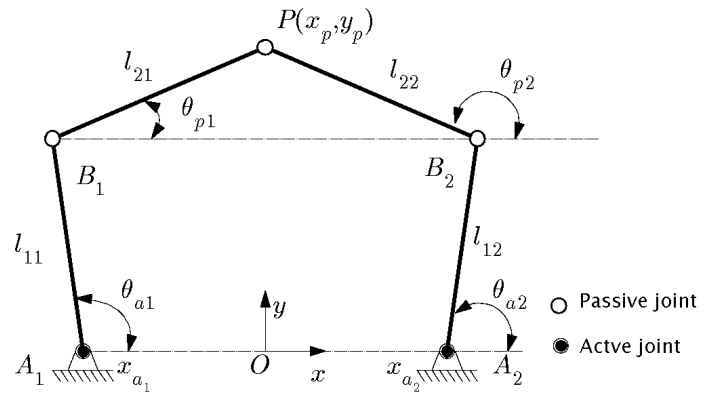

Fig. 1: The 2-DOF Planar Parallel Robot

where $\boldsymbol{\tau}_{i}=\left(\tau_{a i}, \tau_{p i}\right)^{T}$ is the vector of the joint torque, $\mathbf{f}_{i}=\left(f_{a i}, f_{p i}\right)^{T}$ is the vector of the active and passive joint friction, $\boldsymbol{\theta}_{i}=\left(\theta_{a i}, \theta_{p i}\right)^{T}$ is the vector of joints for each $i-t h$ kinematic chain. Moreover, $L_{i}$ is the Lagrangian defined by:

$$
L_{i}=K_{i}-P_{i}
$$

With $K_{i}$ and $P_{i}$ being the kinematic and potential energy, respectively. As the gravity is acting in the $-z$ axis direction, the potential energy is null. Figure 2 shows one of the kinematic chains.

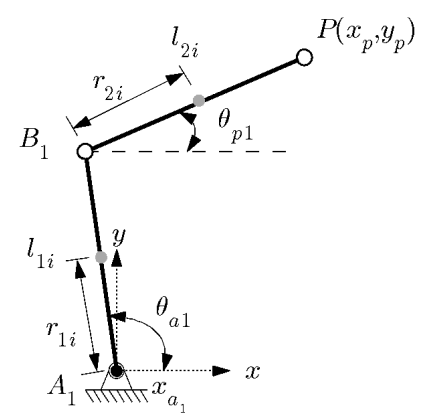

Fig. 2: Kinematic chain of the robot

The total kinetic energy is $K_{i}=K_{1 i}+K_{2 i}$, where $K_{1 i}$ and $K_{2 i}$ represent the kinetic energy of the first and second link of the $i-t h$ kinematic chain respectively (see Fig. 2), thus:

$$
\begin{aligned}
K_{1 i} & =\frac{1}{2} I_{z 1 i} \dot{\theta}_{a i}^{2}+\frac{1}{2} m_{1 i}\left(\dot{x}_{c 1 i}^{2}+\dot{y}_{c 1 i}^{2}\right) \\
K_{2 i} & =\frac{1}{2} I_{z 2 i} \dot{\theta}_{p i}^{2}+\frac{1}{2} m_{2 i}\left(\dot{x}_{c 2 i}^{2}+\dot{y}_{c 2 i}^{2}\right)
\end{aligned}
$$

$m_{1 i}$ and $m_{1 i}$ are the masses, and $I_{z 1 i}$ and $I_{z 1 i}$ are the inertia moments of the links for each $i-t h$ kinematic chain. Additionally, $\left(x_{c 1 i}, y_{c 1 i}\right)^{T}$ and $\left(x_{c 2 i}, y_{c 2 i}\right)^{T}$ are the Cartesian positions of the center of mass of each link that are defined by:
By substituting the previous relations in the Lagrangian of Eq. (2), it is obtained:

$$
L_{i}=K_{i}=\frac{1}{2} \dot{\theta}_{a i}^{2} \alpha+\frac{1}{2} \dot{\theta}_{p i}^{2} \beta+\gamma \dot{\theta}_{a i} \dot{\theta}_{p i} \cos \left(\theta_{a i}-\theta_{p i}\right)
$$

where $\alpha_{i}=I_{z 1 i}+m_{1 i} r_{1 i}^{2}+m_{2 i} L_{1 i}^{2}, \beta_{i}=I_{z 2 i}+m_{2 i} r_{2 i}^{2}$ and $\gamma_{i}=m_{2 i} L_{1 i} r_{2 i}$.

The dynamics of each $i-t h$ kinematic chain is obtained by substituting the Lagrangian of Eq. (5) into the Lagrange equation of Eq. (1), thus:

$$
\boldsymbol{\tau}_{i}-\mathbf{f}_{i}=\mathbf{M}_{i}\left(\boldsymbol{\theta}_{i}\right) \ddot{\boldsymbol{\theta}}_{i}+\mathbf{C}_{i}\left(\boldsymbol{\theta}_{i}, \dot{\boldsymbol{\theta}}_{i}\right) \dot{\boldsymbol{\theta}}_{i}
$$

where

$\mathbf{M}_{i}\left(\boldsymbol{\theta}_{i}\right)=\left[\begin{array}{cc}\alpha_{i} & \gamma_{i} \cos \left(\theta_{a i}-\theta_{p i}\right) \\ \gamma_{i} \cos \left(\theta_{a i}-\theta_{p i}\right) & \beta_{i}\end{array}\right]$
$\mathbf{C}_{i}\left(\boldsymbol{\theta}_{i}, \dot{\boldsymbol{\theta}}_{i}\right)=\left[\begin{array}{cc}0 & \gamma_{i} \dot{\theta}_{p i} \sin \left(\theta_{a i}-\theta_{p i}\right) \\ -\gamma_{i} \dot{\theta}_{a i} \sin \left(\theta_{a i}-\theta_{p i}\right) & 0\end{array}\right]$

\section{B. Dynamic Model}

The complete dynamic model of the two kinematic chains is formulated by combining the model of the two kinematic chains of Eq. (6), thus:

$$
\mathbf{M}(\boldsymbol{\theta}) \ddot{\boldsymbol{\theta}}+\mathbf{C}(\boldsymbol{\theta}, \dot{\boldsymbol{\theta}}) \dot{\boldsymbol{\theta}}+\mathbf{f}=\boldsymbol{\tau}
$$

with $\boldsymbol{\theta}=\left(\boldsymbol{\theta}_{a}, \boldsymbol{\theta}_{p}\right)^{T}, \dot{\boldsymbol{\theta}}=\left(\dot{\boldsymbol{\theta}}_{a}, \dot{\boldsymbol{\theta}}_{p}\right)^{T}, \mathbf{f}=\left(\mathbf{f}_{a}, \mathbf{f}_{p}\right)^{T}$ and $\boldsymbol{\tau}=$ $\left(\boldsymbol{\tau}_{a}, \boldsymbol{\tau}_{p}\right)^{T} \cdot \boldsymbol{\tau}_{a}=\left(\tau_{a 1}, \tau_{a 2}\right)^{T}$ is the vector of input torque in the active joints, $\boldsymbol{\tau}_{p}=\left(\tau_{p 1}, \tau_{p 2}\right)^{T}$ is the vector of the torque in the passive joints . $\mathbf{f}_{a}=\left(f_{a 1}, f_{a 2}\right)^{T}$ is the vector of frictions in the active joints, $\mathbf{f}_{p}=\left(f_{p 1}, f_{p 2}\right)^{T}$ is the vector of frictions in the passive joints. An input torque is not applied in passive joints, thus $\boldsymbol{\tau}_{p}=(0,0)^{T}$. Moreover, the friction in passive joints is assumed negligible when compared with friction in active joints thus $\mathbf{f}_{p}=(0,0)^{T}$. The friction in the active joints considers the viscus friction, $v_{i} \dot{\theta}_{a i}$, and coulomb friction, $d_{i} \operatorname{sign}\left(\dot{\theta}_{a i}\right)$, thus:

$$
f_{a i}=v_{i} \dot{\theta}_{a i}+d_{i} \operatorname{sign}\left(\dot{\theta}_{a i}\right)
$$

The mass and Coriolis matrices (see Eq. (7)) of both kinematic chains are given by:

$$
\begin{gathered}
\mathbf{M}(\boldsymbol{\theta})=\left[\begin{array}{cccc}
\alpha_{1} & 0 & \gamma_{1} c_{a p 1} & 0 \\
0 & \alpha_{2} & 0 & \gamma_{2} c_{a p 2} \\
\gamma_{1} c_{a p 1} & 0 & \beta_{1} & 0 \\
0 & \gamma_{2} c_{a p 2} & 0 & \beta_{2}
\end{array}\right] \\
\mathbf{C}(\boldsymbol{\theta}, \dot{\boldsymbol{\theta}})=\left[\begin{array}{cccc}
0 & 0 & \gamma_{1} \dot{\theta}_{p 1} s_{a p 1} & 0 \\
0 & 0 & 0 & \gamma_{2} \dot{\theta}_{p 2} s_{a p 2} \\
-\dot{\theta}_{1} \dot{\theta}_{a 1} s_{a p 1} & 0 & 0 & 0 \\
0 & -\gamma_{2} \dot{\theta}_{a 2} s_{a p 2} & 0 & 0
\end{array}\right]
\end{gathered}
$$

where $c_{a p i}=\cos \left(\theta_{a i}-\theta_{p i}\right), s_{a p i}=\sin \left(\theta_{a i}-\theta_{p i}\right)$, for $i=$ 1,2 . 


\section{Complete dynamic model}

The complete dynamic model is obtained by considering the coupling of the kinematic chains at the passive joint of point $P$. The kinematic constraints of the coupling are derived from the Jacobian matrix. By using the D'Alembert's principle and the principle of virtual work, the torques of the active joint $\tau_{a}$ and torque of the joints $\tau$ satisfy the relation:

$$
\tau_{a}=\Psi^{T} \boldsymbol{\tau}
$$

where $\Psi=\partial \boldsymbol{\theta} / \partial \boldsymbol{\theta}_{a}$, consequently $\Psi=[\mathbf{I}, \mathbf{J}]$ with $\mathbf{I}$ being the identity matrix and $\mathbf{J}=\partial \boldsymbol{\theta}_{p} / \partial \boldsymbol{\theta}_{a}$ where:

$$
\mathbf{J}=\frac{\partial \boldsymbol{\theta}_{p}}{\partial \boldsymbol{\theta}_{a}}=-\left[\frac{\partial \mathbf{h}}{\partial \boldsymbol{\theta}_{p}}\right]^{-1}\left[\frac{\partial \mathbf{h}}{\partial \boldsymbol{\theta}_{a}}\right]
$$

with:

$$
\mathbf{h}=\left[\begin{array}{c}
l_{11} c_{a 1}+l_{12} c_{p 1}+x_{a 1}-l_{21} c_{a 2}-l_{22} c_{p 2}-x_{a 2} \\
l_{11} s_{a 1}+l_{12} s_{p 1}-l_{21} s_{A 2}-l_{22} s_{p 2}
\end{array}\right]
$$

where $c_{a i}=\cos \theta_{a i}, s_{a i}=\sin \theta_{a i}, c_{p i}=\cos \theta_{p i}, s_{p i}=$ $\sin \theta_{p i}$ for $i=1,2$. The dynamic equation of Eq. (7) is multiplied by the constraint of Eq. (9) leading to:

$$
\Psi^{T} \mathbf{M}(\boldsymbol{\theta}) \ddot{\boldsymbol{\theta}}+\Psi^{T} \mathbf{C}(\boldsymbol{\theta}, \dot{\boldsymbol{\theta}}) \dot{\boldsymbol{\theta}}+\Psi^{T} \mathbf{f}=\Psi^{T} \boldsymbol{\tau}
$$

Moreover, the following relations are established:

$$
\begin{aligned}
\dot{\boldsymbol{\theta}} & =\frac{\partial \boldsymbol{\theta}}{\partial \boldsymbol{\theta}_{a}} \dot{\boldsymbol{\theta}}_{a} \\
\ddot{\boldsymbol{\theta}} & =\dot{\Psi} \dot{\boldsymbol{\theta}}_{a}+\Psi \ddot{\boldsymbol{\theta}}_{a}
\end{aligned}
$$

The relation of Eqs. (9), (12) and (13) are substituted into Eq. (11), therefore the total dynamic equation is expressed as follows:

$$
\mathbf{M}_{t} \ddot{\boldsymbol{\theta}}_{a}+\mathbf{C}_{t} \dot{\boldsymbol{\theta}}_{a}+\mathbf{f}_{a}=\boldsymbol{\tau}_{a}
$$

where $\mathbf{M}_{t}=\Psi^{T} \mathbf{M}(\boldsymbol{\theta}) \Psi$ and $\mathbf{C}_{t}=\Psi^{T} \mathbf{M}(\boldsymbol{\theta}) \dot{\Psi}+$ $\Psi^{T} \mathbf{C}(\boldsymbol{\theta}, \dot{\boldsymbol{\theta}}) \Psi$.

\section{Position tracking control}

Computed Torque Control (CTC) is composed of two independent loops: an inner-loop to linearize the non-linear dynamic of the robot by means of feedback linearization and an outer-loop to track a desired trajectory. Thus, the nonlinear dynamic equation of the robot (Eq. (14)) can be written in a simplified way:

$$
\boldsymbol{\tau}_{a}=\mathbf{A}(\boldsymbol{\theta}) \ddot{\boldsymbol{\theta}}_{a}+\mathbf{h}(\boldsymbol{\theta}, \dot{\boldsymbol{\theta}})
$$

where $\mathbf{A}(\boldsymbol{\theta})=\mathbf{M}_{t}$ and $\mathbf{h}(\boldsymbol{\theta}, \dot{\boldsymbol{\theta}})=\mathbf{C}_{t}+\mathbf{f}_{a}$.

The robot equations can be linearized and decoupled by nonlinear feedback. Let $\hat{\mathbf{A}}(\boldsymbol{\theta})$ and $\hat{\mathbf{h}}(\boldsymbol{\theta}, \dot{\boldsymbol{\theta}})$ be the estimates of $\mathbf{A}(\boldsymbol{\theta})$ and $\mathbf{h}(\boldsymbol{\theta}, \dot{\boldsymbol{\theta}})$, respectively. Assuming that:

$$
\hat{\mathbf{A}}(\boldsymbol{\theta})=\mathbf{A}(\boldsymbol{\theta}) \quad \hat{\mathbf{h}}(\boldsymbol{\theta}, \dot{\boldsymbol{\theta}})=\mathbf{h}(\boldsymbol{\theta}, \dot{\boldsymbol{\theta}})
$$

the problem is reduced to a $n$ linear and decoupled doubleintegrator system, where $n$ is the number of degrees of freedom of the robot (i.e. $n=2$ in this application).

$$
\ddot{\boldsymbol{\theta}}=\mathbf{w}_{c}
$$

with $\mathbf{w}_{c}$ being the new input control vector. Equation (16) corresponds to the inverse dynamic control scheme, where the dynamic of the robot is transformed into a set of double integrators (see Fig. 3).

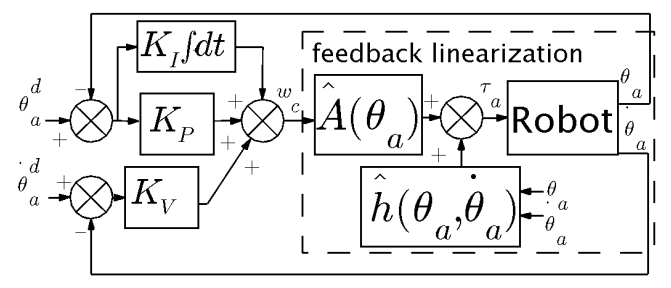

Fig. 3: The computed torque and PID block diagram.

Considering a PID controller on each joint in the outer loop (assuming perfect decoupling between the axes) one has:

$$
\mathbf{w}_{c}=\mathbf{K}_{P}\left(\boldsymbol{\theta}_{a}^{d}-\boldsymbol{\theta}_{a}\right)+\mathbf{K}_{D}\left(\dot{\boldsymbol{\theta}}_{a}^{d}-\dot{\boldsymbol{\theta}}_{a}\right)+\mathbf{K}_{I} \int\left(\boldsymbol{\theta}_{a}^{d}-\boldsymbol{\theta}_{a}\right) d t
$$

The controller gains are $\mathbf{K}_{P}=\operatorname{diag}\left(k_{P_{1}}, k_{P_{2}}\right), \mathbf{K}_{D}=$ $\operatorname{diag}\left(k_{D_{1}}, k_{D_{2}}\right)$ and $\mathbf{K}_{I}=\operatorname{diag}\left(k_{I_{1}}, k_{I_{2}}\right)$. It is worth to mention that the joint space position error is $\mathbf{e}=\boldsymbol{\theta}_{a}^{d}-\boldsymbol{\theta}_{a}$, where $\mathbf{e}=\left(e_{1}, e_{2}\right)^{T} \in \mathbb{R}^{2 \times 1}$. The controller gains are tuned in order to have in continuous-time domain the following closed-loop characteristic equation for each decoupled double-integrator of Eq. (16): $\left(s+\omega_{r}\right)\left(s^{2}+2 \xi \omega_{r} s+\omega_{r}^{2}\right)=0$, where $s$ is the Laplace variable. By selecting $\xi=1$ all the closed-loop poles are assigned to $\omega_{r}$. Thus $k_{P}=(1+2 \xi) \omega_{r}^{2}$, $k_{D}=(1+2 \xi) \omega_{r}, k_{I}=\omega_{r}^{3}$, additional details can be obtained in [7].

For the position tracking control scheme shown in Fig. 3, it was assumed in Eq. (16) that the estimated and real terms are equal. Experimentally, this assumption is not valid due to the existence of unknown or uncertain parameters, e.g. friction parameters. In this way, it is necessary to analyze the effects of uncertain frictions on the closed-loop dynamics of the robot system. The parameters of the friction are defined by the vector of parameters $\mathrm{x}$, where

$$
\mathbf{x}=\left[\begin{array}{llll}
v_{1} & v_{2} & d_{1} & d_{2}
\end{array}\right]^{T}
$$

\section{ANALysis OF DyNAmic Systems UNDER FuZzY PARAMETERS}

In several cases, some parameters of the system cannot be accurately estimated due to their small variations around their nominal values. In these cases, these parameters can be modeled by means of fuzzy variables. The fuzzy set theory was initially formulated by [8] to represent vague or ambiguous information. Thereby, it is possible to represent inaccurate or uncertain parameters by using fuzzy variables, specially when the stochastic process which models the uncertain parameters is unknown.

A review of the literature focused on the non-probalistic approaches to analyze parameter uncertainty in which two main approaches where presented to model the uncertainties: 


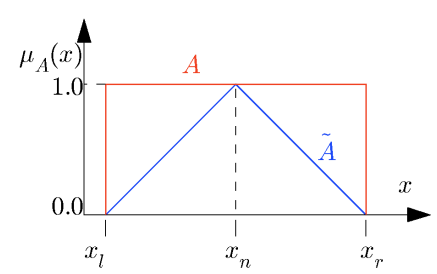

(a)

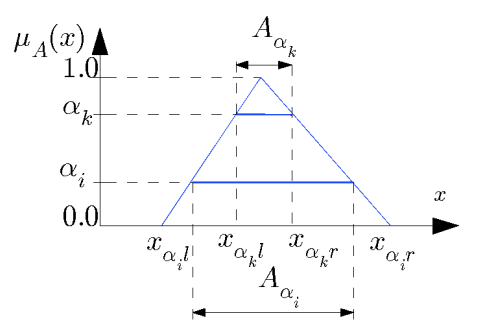

(b)

Fig. 4: Fuzzy sets and $\alpha$-level representation: (a) $\alpha$-levels, (b) Fuzzy set.

interval quantities analysis and fuzzy variables analyis [9]. These two approaches require the solution of interval problems. The methodology to analyze the fuzzy uncertainties in this paper is based on the $\alpha$-level optimization which allows to map fuzzy uncertainties without special properties [10]. The basic concepts of the fuzzy variables are introduced in the bellow.

\section{A. Fuzzy Variables}

Let $\mathbf{X}$ be an universal classical set of objects whose generic elements are denoted by $x$. The subset $A$ (where $A \in \mathbf{X}$ ) is defined by the classical membership function $\mu_{A}: \mathbf{X} \rightarrow\{0,1\}$ (see Fig. 4a). Furthermore, a fuzzy set $\tilde{A}$ is defined by means of the membership function $\mu_{A}: \mathbf{X} \rightarrow[0,1]$, where $[0,1]$ is a continuous interval. The membership function indicates the degree of compatibility of the element $x$ to $\tilde{A}$. The closer the value of $\mu_{A}(x)$ is to " 1 ", the more $x$ belongs to $\tilde{A}$.

Thus, the fuzzy set is completely defined by:

$$
\tilde{A}=\left\{\left(x, \mu_{A}(x)\right) \mid x \in \mathbf{X}\right\} ; 0 \leq \mu_{\mathbf{A}} \leq 1
$$

Fig. 4a presents a fuzzy set described as a fuzzy triangular number, this is the simplest representation of a fuzzy variable that is defined by $x_{l}, x_{r}$ and $x_{n}$. In this method, the fuzzy variables should be represented as a set of intervals for computational purposes by use of the $\alpha$-levels (See Fig. 4b). The fuzzy set $\tilde{A}$ is represented by means of subsets which correspond to real and continuous intervals, thus:

$$
A_{\alpha_{k}}=\left\{x \in \mathbf{X}, \mu_{\mathbf{A}}(x) \geq \alpha_{k}\right\}
$$

The $\alpha$-level subsets of $\tilde{A}$ have the property:

$$
\underline{A}_{\alpha_{k}} \subseteq \underline{A}_{\alpha_{i}} \forall \alpha_{i}, \alpha_{k} \in(0,1] \quad ; \quad \alpha_{i} \leq \alpha_{k}
$$

If the fuzzy set is convex (in the unidimensional case), each $\alpha$-level subset $A_{\alpha_{k}}$ corresponds to the interval $\left[x_{\alpha_{k} l}, x_{\alpha_{k} r}\right]$ (see Fig. 4b), where:

$$
\begin{aligned}
& x_{\alpha_{k} l}=\min \left[x \in \mathbf{X} \mid \mu_{A}(x) \geq \alpha_{k}\right] \\
& x_{\alpha_{k} r}=\max \left[x \in \mathbf{X} \mid \mu_{A}(x) \geq \alpha_{k}\right]
\end{aligned}
$$

\section{B. Fuzzy Dynamic Analysis}

The fuzzy dynamic analysis is an appropriate method to map a fuzzy input vector $\underline{\tilde{x}}$ onto the output $\underline{\tilde{z}}$ using the numerical model of the parallel robot. The fuzzy dynamic analysis based on the $\alpha$-optimization [11] is mainly composed of two stages illustrated in Fig. 5.

In the first stage, for computational purposes, the input vector that corresponds to the fuzzy parameters is discretized by means of the $\alpha$-level representation used in Eq. (20) and Fig. 4b. Thus, each element of the fuzzy parameters vector $\underline{\tilde{\mathbf{x}}}=\left(\tilde{x}_{1}, \ldots, \tilde{x}_{4}\right)$ is considered as an interval $X_{i \alpha_{k}}=\left[x_{i \alpha_{k} l}, x_{i \alpha_{k} r}\right]$, where $\alpha_{k} \in(0,1]$ and $i=1, \ldots, 4$. Consequently, the crisp sub-space $\underline{X}_{\alpha_{k}}$ is defined, so that $\underline{X}_{\alpha_{k}}=\left(X_{1 \alpha_{k}}, \ldots, X_{4 \alpha_{k}}\right)$, where $\underline{X}_{\alpha_{k}}$ considers the four uncertain parameters of Eq. (18).

The second stage is related to solving an optimization problem. This optimization problem consists in finding the maximum and minimum value of the output for the mapping model given by $\underline{z}=f(\underline{x})$ where $f(\underline{x})$ is the dynamic model as function of the set of parameters $\underline{x}$, thus:

$$
z_{\alpha_{k} r}=\max _{\underline{x} \in \underline{X}_{\alpha_{k}}} \mathbf{f}(\underline{x}) \quad z_{\alpha_{k} l}=\min _{\underline{x}_{\in} \underline{X}_{\alpha_{k}}} \mathbf{f}(\underline{x})
$$

$z_{\alpha_{k} r}$ and $z_{\alpha_{k} l}$ correspond to the upper and lower bounds of the interval $\left[z_{\alpha_{k} r}, z_{\alpha_{k} l}\right]$ in the $\alpha$-level $\alpha_{k}$. The set of discretized intervals $\left[z_{\alpha_{k} r}, z_{\alpha_{k} l}\right]$ for $\alpha_{k} \in(0,1]$ forms the whole fuzzy resulting variable $\tilde{z}$ (see Fig. 5).

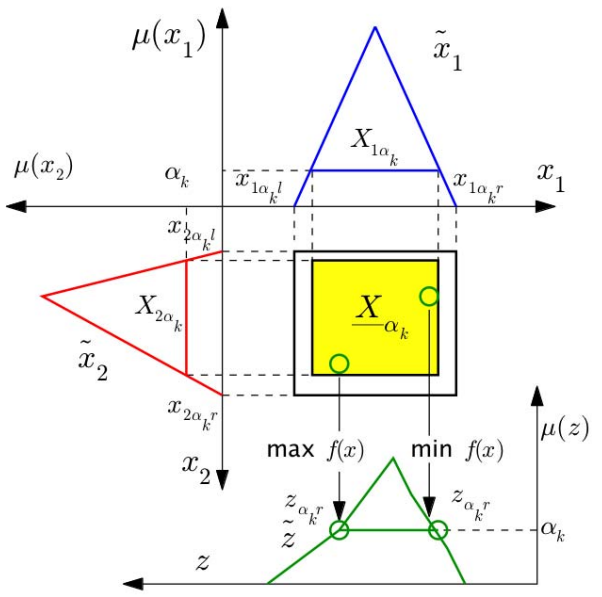

Fig. 5: Sub-space $\underline{X}_{\alpha_{k}}$ and resulting fuzzy variable $\tilde{z}$.

The fuzzy dynamic analysis of a transient time-domain system demands the solution of a large number of optimization problems regarding all $\alpha$-level of interest for each 
considered time step. Each upper and lower bound of the system analysis at a certain time instant is obtained by using Differential Evolution algorithm [12] since evolutionary strategies have been used to solve optimization problems in robotics with success [13]. The output value of the transient analysis at the evaluated time-step constitutes the objective function of the optimization problem. The inputs to this function are the uncertain parameters previously described in Eq. (18). Full developments of the method are given in [10].

\section{Simulation Results}

The model of the controlled robot of Fig. 3 was used in the numerical simulation that was implemented in software matlab $\AA /$ simulink. The values of parameters of the parallel robot are given in table I. The gains of the controller are determined by selecting the parameters $\xi=1$ and $\omega_{r}=$ $70 \mathrm{rad} / \mathrm{s}$ in order to obtain a damped response without overshoot.

TABLE I: Parameters of the robot.

\begin{tabular}{|c|c||c|c|}
\hline Parameter & Value & Parameter & Value \\
\hline$l_{11}(\mathrm{~m})$ & 0.244 & $I_{z 12}\left(\mathrm{~kg} / \mathrm{m}^{2}\right)$ & 0.010 \\
$l_{12}(\mathrm{~m})$ & 0.244 & $I_{z 21}\left(\mathrm{~kg} / \mathrm{m}^{2}\right)$ & 0.005 \\
$l_{21}(\mathrm{~m})$ & 0.244 & $I_{z 22}\left(\mathrm{~kg} / \mathrm{m}^{2}\right)$ & 0.005 \\
$l_{22}(\mathrm{~m})$ & 0.244 & $v_{1}(\mathrm{Nms} / \mathrm{rad})$ & 100 \\
$m_{a i}(\mathrm{~kg})$ & 1.2 & $v_{2}(\mathrm{Nms} / \mathrm{rad})$ & 100 \\
$m_{b i}(\mathrm{~kg})$ & 1.0 & $d_{1}(\mathrm{Nm})$ & 50 \\
$r_{1 i}(\mathrm{~m})$ & 0.12 & $d_{2}(\mathrm{Nm})$ & 50 \\
$r_{2 i}(\mathrm{~m})$ & 0.12 & $x_{a 1}(\mathrm{~m})$ & 0.25 \\
$I_{z 11}\left(\mathrm{Kgm}^{2}\right)$ & 0.010 & $x_{a 2}(\mathrm{~m})$ & 0.25 \\
\hline
\end{tabular}

The uncertain parameters are modeled by means of fuzzy triangular numbers which is the simplest manner to describe a fuzzy variable, the triangular fuzzy variable was previously described in Fig. 4a. Hence this representation of the fuzzy uncertain parameters is sufficient for the numerical evaluation of an uncertain parameter in the fuzzy dynamic analysis. Thus, a fuzzy variable can be written as:

$$
\tilde{x}_{i}=x_{i}(1-p / 100,1,1+p / 100)
$$

where $x_{i}$ represents the nominal value of the parameter $i t h$ parameter of $\mathbf{x}$ of Eq. (18) and $p$ stands for the maximum percentage of dispersion in level $\alpha=0$. In the analysis, the fuzzy response is evaluated considering three different $\alpha$ levels as a means to evaluate the $\alpha=$ level in the maximum, minimum and intermediate value. When $\alpha=0$ the major dispersion is considered, $\alpha=0.5$ considers an intermediate level of uncertainty and $\alpha=1$ evaluates the response with the nominal parameters.

The parameters used in the Differential Evolution Algorithm to solve the $\alpha$-level optimization of the fuzzy analysis are given as follows: population size $=10$ per uncertain variable, 100 generations, crossover probability rate $=0.8$, perturbation rate $=0.8$, and the strategy for the mutation mechanism is DE/RAND/1/BIN. These parameters have been successfully tested in various previous contributions [12]. The objective function for the $\alpha$-level optimization is based on the joint space position error e $(t)$ (see Fig 3), thus:

$$
f(\underline{\tilde{\mathbf{x}}}, k)=\left|e_{1}(k)\right|+\left|e_{2}(k)\right|
$$

with $\underline{x}$ being the set of the considered uncertain parameters at a specific discrete step time $k$. The tracking trajectory is analyzed to evaluate the effect of small uncertain friction parameters on the position accuracy.

In order to analyze the effect of uncertainties in the position accuracy of the robot, small variations in some parameters around their nominal values are introduced in the model. Table II gives the maximum percentage of dispersion of each triangular fuzzy parameter considered in the model. The dispersion of the structural parameters are in agreement with standard manufacturing tolerances.

TABLE II: Dispersion of Fuzzy uncertain parameters.

\begin{tabular}{|c|c||c|c|}
\hline Parameter & Dispersion $(\mathrm{p})$ & Parameter & Dispersion $(\mathrm{p})$ \\
\hline$v_{1}$ & $5 \%$ & $d_{1}$ & $5 \%$ \\
$v_{1}$ & $5 \%$ & $d_{2}$ & $5 \%$ \\
\hline
\end{tabular}

A circular workspace reference trajectory with translational motion of the movable platform in $x-y$ axis was implemented (Fig. 6).

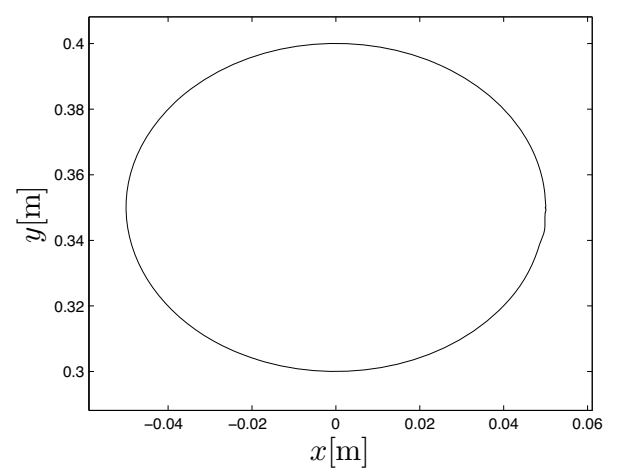

Fig. 6: Workspace reference, circular trajectory.

The joint space reference trajectories for the two active joints are generated from the circular workspace trajectory of Fig. 6 with the help of the inverse kinematic model. As a result, the reference trajectories for the two active joints are obtained in Fig. 7.

Fig. 8 shows the tracking error of the joints of the active joints considering the fuzzy uncertain parameters of table II. Even though the controller was tuned to provide a damped response, the accuracy of the manipulator is affected by small uncertainties on the friction parameters; consequently this variation increases the position error of the parallel robot. As seen, the maximum variation in the joint error over the circular trajectory of each joint is obtained when $\alpha=0$. This variation is not negligible and it should be considered in the overall performance of the parallel robot.

Joint errors are not negligible as seen in Fig. 8. Consequently, the variation of the trajectory of the end effector produced by the uncertain parameters is evaluated in order to 

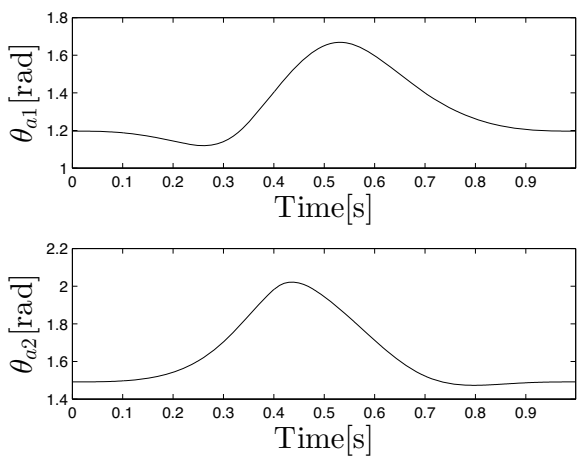

Fig. 7: Joint reference of the active joints.

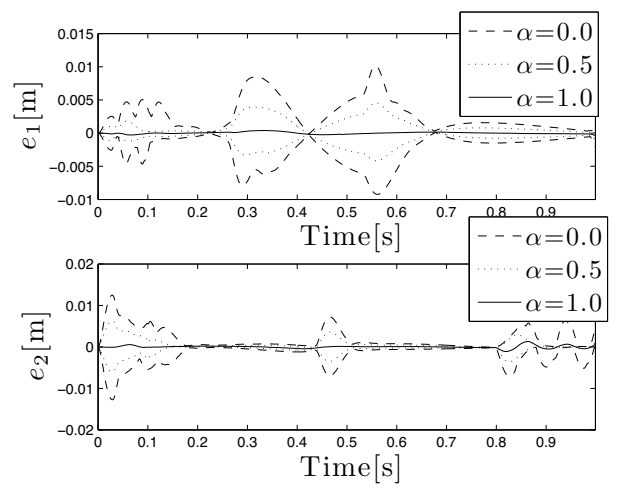

Fig. 8: Joint error, tracking error with fuzzy parameters.

determine how uncertain parameters affect the accuracy. The variation in the position tracking of the end effector in the $x y$-plane is showed in Fig. 9. The results indicate that small variations of the friction parameters produce a significant variation in the tracking position introducing position error during the motion of the end effector.

\section{CONCLUSIONS}

The effect of uncertain parameters on the dynamic behavior of a 2-DOF fully parallel robot was analyzed in this contribution. Specifically, the PID computed torque was analyzed by considering uncertain frictions in the joints. The simulation results indicate that small uncertain friction in the active joints can affect significantly the dynamic behavior of the system in terms of position accuracy. Therefore, uncertain parameters should be taken into account in numerical simulation to obtain reliable numerical models for design purposes.

The fuzzy dynamic analysis used in this contribution demonstrated to be a straightforward methodology to quantify the effect of uncertain parameters on the dynamic response of a parallel robot manipulator.

Further work will encompass the study of control techniques and design methods of parallel robots under uncertain parameters and dynamics.

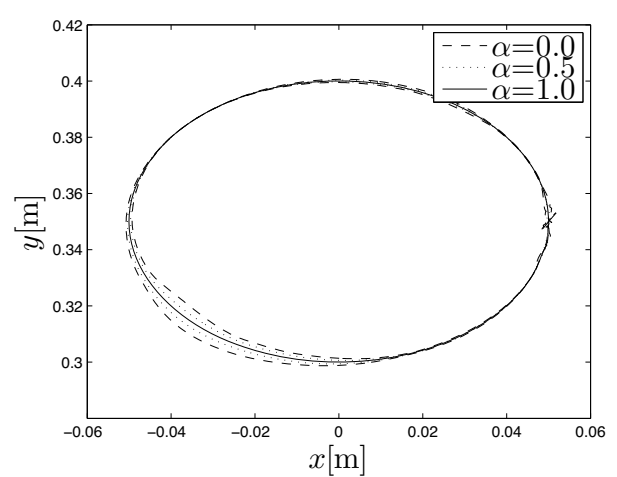

Fig. 9: Tracking position of the end effector subject to uncertain friction in $x y$-plane.

\section{ACKNOWLEDGMENT}

The authors express their acknowledgements to the Graduate Program in Mechanical Engineering of the Federal University of Technology - Paraná funded by CAPES.

\section{REFERENCES}

[1] J. Kim, W.-J. Song, and B.-S. Kang, "Stochastic approach to kinematic reliability of open-loop mechanism with dimensional tolerance," Applied Mathematical Modelling, vol. 34, pp. 1225-1237, 2010.

[2] M. D. Pandey and X. Zhang, "System reliability analysis of the robotic manipulator with random joint clearances," Mechanism and Machine Theory, vol. 58, pp. 137-152, 2012.

[3] P. Voglewede, A. H. C. Smith, and A. Monti, "Dynamic performance of a scara robot manipulator with uncertainty using polynomial chaos theory," IEEE Transactions on Robotics, vol. 25, no. 1, pp. 206-210, 2009.

[4] F. Lara-Molina, E. Koroishi, and V. Steffen, "Uncertainty analysis of a two-link robot manipulator under fuzzy parameters," in Robotics: SBRLARS Robotics Symposium and Robocontrol (SBR LARS Robocontrol), 2014 Joint Conference on, pp. 1-6, Oct 2014.

[5] F. A. Lara-Molina, E. H. Koroishi, and V. Steffen Jr., "Análise estrutural considerando incertezas paramétricas fuzzy," in Técnicas de Inteligtncia Computacional com Aplicações em Problemas Inversos de Engenharia (F. S. Lobato, V. Steffen Jr., and A. J. da Silva Neto, eds.), ch. 11, pp. 133-144, Curitiba, PR: Omnipax, 1 ed., 2014

[6] T. D. Le, H.-J. Kang, and Y.-S. Suh, "Chattering-free neuro-sliding mode control of 2-dof planar parallel manipulators," International Journal of Advanced Robotic Systems, vol. 10, pp. 104-130, 2013.

[7] F. A. Lara-Molina, J. M. Rosário, D. Dumur, and P. Wenger, "Application of predictive control techniques within parallel robot," Sba: Controle \& Automação Sociedade Brasileira de Automatica, vol. 23, pp. $530-540,2012$

[8] L. Zadeh, "Fuzzy sets," Information and Control, vol. 8, pp. 338-353, 1965

[9] D. Moens and M. Hanss, "Non-probabilistic finite element analysis for parametric uncertainty treatment inapplied mechanics: Recent advances," Finite Elementsin Analysis and Design, vol. 47, no. 1, p. 2011, 2011 .

[10] B. Möller and M. Beer, Fuzzy Randomness, Uncertainty in Civil Engineering and Computational Mechanics. Springer-Verlag, 2004.

[11] B. Möller, W. Graf, and M. Beer, "Fuzzy structutal analysis using $\alpha$ level optimizaton," Computational Mechanics, vol. 26, pp. 547-565, 2000.

[12] K. V. Price, R. M. Storn, and J. A. Lampinen, Differential evolution a practical approach to global optimization. Springer-Verlag, 2005.

[13] F. A. Lara-Molina, J. M. Rosário, and D. Dumur, "Multi-objective optimization of stewart-gough manipulator using global indices," in Advanced Intelligent Mechatronics (AIM), 2011 IEEE/ASME International Conference on, pp. 79-85, 2011. 